\title{
Maltrato Infantil: Conocimiento y Actitud del Cirujano Dentista. Marco Legal y Revisión de la Literatura
}

\author{
Child Abuse: Knowledge and Attitude of the Dentist. \\ Legal Framework and Review of the Literature
}

\author{
Carla Cifuentes Harris ${ }^{1}$; Claudia Contreras Reyes²; María Angélica Torres ${ }^{1}$ \& Sebastián Gonzales Alegría ${ }^{3}$
}

CIFUENTES, H. C.; CONTRERAS, R. C.; TORRES, M. A. \& GONZALES, A. S. Maltrato infantil: conocimiento y actitud del cirujano dentista. Marco legal y revisión de la literatura. Int. J. Odontostomat., 14(2):160-166, 2020.

RESUMEN: El maltrato, abuso y la negligencia a los niños constituyen un fenómeno social que desafortunadamente está extendido por todo el mundo sin barreras étnicas, geográficas ni de contexto social. En Chile, hasta el año 2012 el 71 $\%$ de los niños sufría algún tipo de maltrato al interior de su familia. Las lesiones cráneo-faciales y de cuello ocurren en más de la mitad de los casos. El Odontólogo tiene la obligación legal, moral y ética como ciudadano y profesional de la salud de notificar a las autoridades competentes los casos sospechosos de maltrato. Objetivo: Evaluar cuál es el conocimiento del cirujano dentista sobre maltrato infantil y cuál ha sido su actitud frente a la detección y reporte del maltrato infantil. Búsqueda bibliográfica en PUBMED utilizando palabras clave: "child abuse" and "dentistry". Se encontraron 132 artículos, de los cuales fueron 15 atingentes con texto completo. Estudios realizados en varios países informaron de las dificultades de los odontólogos en el diagnóstico, la documentación y la denuncia de casos con sospecha de abuso a las autoridades, además de la necesidad de mayor formación en el tema. Dada la importancia del tema, el papel del odontólogo al trabajar directamente con niños y adolescentes, y el aumento en el reporte de casos sospechosos de violencia, se sugiere fuertemente realizar una vigilancia respecto a conocimiento y actitudes entre los odontólogos en Chile.

PALABRAS CLAVE: maltrato infantil, odontología, marco legal.

\section{INTRODUCCIÓN}

El maltrato, abuso y la negligencia a los niños constituyen un fenómeno social que desafortunadamente está extendido por todo el mundo sin barreras étnicas, geográficas ni de contexto social, por lo que constituye un problema de salud pública, y los enfoques sistemáticos, basados en la evidencia, multidisciplinarios y sostenibles son las mejores formas de combatirlo (Ministerio de Salud de Chile, 2014).

La UNICEF estima que en América Latina existen, por lo menos, 6 millones de niños maltratados severamente, de los cuales 80 mil mueren anualmente a consecuencia de la violencia, por ello el maltrato infantil es reconocido por OMS/OPS como un problema de salud pública (Krug et al., 2002).

El maltrato infantil es cualquier acción u omisión no accidental que, desde una relación de depen- dencia y/o abuso de poder, cause detrimento en la integridad física, psicológica y social del niño o que amenace su desarrollo físico y psicosocial normal. Se entenderá por acción, la agresión directa, sea ésta física, psicológica o sexual. Se entenderá por omisión, la falta en proporcionar los cuidados tanto físicos como psicológicos que un niño necesita, de acuerdo a su edad y características individuales, de parte de las personas o instituciones que se encuentran obligadas a brindarle protección y cuidado.(Ministerio de Desarrollo Social, 2003).

La UNICEF, por su parte, define como víctima de maltrato y abandono a los niños, niñas y adolescentes hasta los 18 años que sufren ocasional o habitualmente, actos de violencia física, sexual y/o emocional, sea en el grupo familiar o en las instituciones sociales. El maltrato puede ser ejecutado por omisión,

\footnotetext{
${ }^{1}$ Especialidad Odontología Legal y Forense, Universidad de Chile, Santiago, Chile.

${ }^{2}$ Especialista en Odontología Legal y Forense, Servicio Médico Legal, Universidad de Chile, Santiago, Chile.

${ }^{3}$ Residente Ortopedia y Traumatología, Universidad de Chile, Santiago, Chile.
} 
supresión o trasgresión de los derechos individuales y colectivos e incluye el abandono completo o parcial (UNICEF, 2015).

Según el Ministerio de Justicia Chileno el maltrato infantil es cualquier acción u omisión no accidental que, desde una relación de dependencia y/o abuso de poder, cause detrimento en la integridad física, psicológica y social del niño o que amenace su desarrollo físico y psicosocial normal.

La Ley Chilena $N^{\circ} 19.324$ de violencia familiar, lo define como el resultante de una acción u omisión que produzca menoscabo en la salud física o psíquica de los menores. Puede provenir de cualquier ámbito, familiar o extra-familiar, incluyendo el institucional.

Para contar con una medida sobre este fenómeno en Chile la UNICEF realizó en el 2014 el cuarto estudio de maltrato infantil en Chile el cual concluyó que un $62,2 \%$ de los niños/as manifiesta haber vivido situaciones de violencia psicológica en su vida y un $56,1 \%$ el último año; un 50,4 \% señala que ha vivido situaciones de violencia física leve durante su vida y un $43 \%$ durante el último año, mientras que un $28,2 \%$ indica que ha vivido situaciones de violencia física grave en su vida y un $22,1 \%$ el último año (UNICEF).

Para enfrentar este problema se requiere un abordaje multidisciplinario e intersectorial, desde todos los niveles y en todos los ámbitos de atención a la infancia y la adolescencia; y considerando que en Chile la mayoría de las políticas públicas de Salud Oral están dirigidas a estos grupos etarios, se hace necesario poner sobre la mesa cual es el conocimiento del cirujano dentista en esta temática, cual es su actitud frente a la detención y reporte del maltrato infantil, y eventualmente si están capacitados y preparados para hacerlo.

Aspectos legales. Con respecto a las responsabilidades legales es importante recordar que dentro de las obligaciones de los funcionarios públicos se encuentra la de denunciar los hechos que revisten carácter de delito, es así que el Estatuto Administrativo señala que: "Será obligación de cada funcionario denunciar ante el Ministerio Público o ante la policía si no hubiere fiscalía en el lugar en que el funcionario presta servicios, con la debida prontitud, los crímenes o simples delitos y a la autoridad competente los hechos de carácter irregular, especialmente de aquéllos que contravienen el principio de probidad administrativa regulado por la ley No 18.575 (Ministerio de Hacienda, 2005).
El artículo 200 del Código Procesal Penal de Chile, indica: "toda persona a cuyo cargo se encontrare un hospital u otro establecimiento de salud semejante, fuere público o privado, dar en el acto cuenta al fiscal de la entrada de cualquier individuo que tuviere lesiones corporales de significación. La denuncia deber consignar el estado del paciente, describir los signos externos de las lesiones e incluir las exposiciones que hicieren el afectado o las personas que lo hubieren conducido. En ausencia del jefe del establecimiento, dar cuenta el que lo subrogare en el momento del ingreso del lesionado.(Ministerio de Justicia, 2000). El incumplimiento de la obligación prevista en este artículo se castiga con la pena que prevé el artículo 494 del Código Penal (Ministerio de Justicia, 1874).

Por su parte, el artículo 175 letra D, del Código Procesal Penal (C.P.P.) dispone que están obligados a denunciar: "En general, los profesionales en medicina, odontología, química farmacia y de otras ramas relacionadas con la conservación o el restablecimiento de la salud, y los que ejercieren prestaciones auxiliares de ellas, que notaren en una persona o en un cadáver señales de envenenamiento o de otro delito"(Ministerio de Justicia, 2000). El plazo para formular la denuncia es dentro de las veinticuatro horas siguientes al momento en que tomaren conocimiento del hecho criminal (Art. 176 C.P.P.) (Comité Operativo de Intervención en Maltrato Infantil del Hospital Roberto del Río et al., 2010).

Siendo el deber de todo ciudadano el conocimiento de las leyes promulgadas, y de los profesionales de la salud el actuar moral y en beneficio del paciente, el odontólogo no se puede quedar atrás y debe crear conciencia y comprensión de esto, así podrá cumplir con el rol protector y defensor de los derechos del niño, ya que el maltrato infantil compromete la calidad de vida digna de las niñas, niños y adolescentes. Además considerando el contacto regular que los odontólogos tienen con los niños y sus familias y que las lesiones cráneo-faciales y de cuello ocurren en más de la mitad de los casos (65\%) de abuso infantil, los odontólogos se encuentran en una posición favorable para observar un comportamiento anormal, identificar e informar casos sospechosos de abuso, maltrato y abandono de menores (American Academy of Pediatrics Committee on Child Abuse and Neglect and the American Academy of Pediatric Dentistry, 2010).

El objetivo del estudio fue conocer, a través de una revisión bibliográfica, cuál es el conocimiento que tienen los cirujanos dentista con respecto a la temáti- 
ca de maltrato infantil, cuál ha sido su participación autoreportada en la detección y reporte del maltrato infantil y si se sienten capacitados teóricamente para hacerlo.

\section{MATERIAL Y MÉTODO}

Se realizó una búsqueda bibliográfica en PUBMED utilizando como palabras clave: "child abuse" and "dentistry", aplicando como filtro: 7 años, con abstract, en humanos e idioma inglés.

\section{RESULTADOS}

En PUBMED se encontraron 132 artículos, de los cuales 16 fueron atingentes, pero solo se tuvo acceso al texto completo de 15 . Del total de artículos solo 2 fueron realizados en Chile (Fig. 1).

En la Tabla I se indican los autores y año de publicación de los 15 estudios analizados, sus países de origen, muestra y quienes fueron los participantes. El promedio de participantes fue de 474 personas, con un mínimo de 58 y un máximo de 1200. Del total de los estudios, 3 fueron realizados con estudiantes de odontología, 9 con cirujanos dentista y 3 con cirujanos dentista e higienistas dentales.

En la Tabla II se observa que el método utilizado para obtener los datos, en 93,3\%(14) de los estudios, fue la encuesta. En cuanto a si los participantes

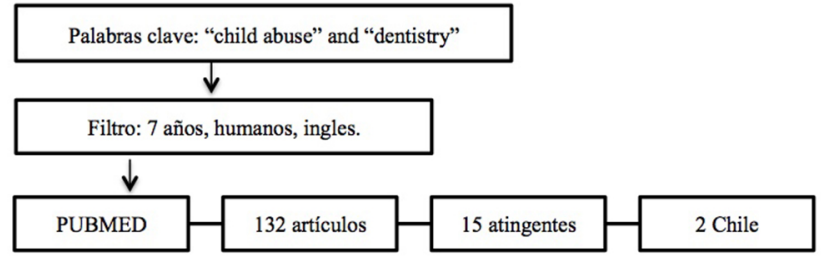

recibieron alguna capacitación o entrenamiento en el tema, no se puede efectuar una buena comparación entre sí esta fue en pregrado o una vez graduados, debido a que este ámbito no fue medido en todos los estudios, pero en los que sí se midió se observa que no hay una tendencia marcada, siendo bastante pareja la distribución de las respuestas, con un promedio de 30,97\% para quienes recibieron la capacitación en pre grado y $35,87 \%$ quienes la recibieron luego de graduarse. Finalmente el $100 \%$ de los encuestados, a los que se les consultó, considera que sí necesita profundizar su capacitación o entrenamiento en el tema en cuestión.

Al evaluar porcentualmente cuantos de los encuestados tuvieron sospechas de estar frente a un posible maltrato o abuso infantil y cuantos lo reportaron, en promedio fueron $35 \%$ y $24 \%$ respectivamente. Sin embargo se advierte que en todos los estudios el porcentaje de sospechas supera al de los reportes. Finalmente se observan cuales fueron las principales razones de no reportar, siendo las principales: dudas en el diagnóstico, miedo a consecuencias negativas para el niño y falta de conocimiento sobre procedimientos de notificación y referencia a los organismos pertinentes (Tabla II).

Tabla I. Análisis bibliográfico (parte 1).

\begin{tabular}{cllcc}
\hline$N^{\circ}$ & Autores y año de publicación & País de origen & Muestra & Participantes \\
\hline 1 & Jordan \& Welbury, 2012 & Croacia & 544 & $\mathrm{E}$ \\
2 & Fierro \& Salazar, 2012 & Chile & 58 & $\mathrm{O}$ \\
3 & Laud et al., 2012 & Grecia & 368 & $\mathrm{O}$ \\
4 & Harris et al., 2013 & Escocia & 628 & $\mathrm{O}$ \\
5 & Cucovic-Bagic \& Welbury, 2014 & Croacia & 510 & $\mathrm{O}$ \\
6 & Tilvawala et al., 2014 & Nueva Zelanda & 320 & $\mathrm{O}$ \\
7 & Asfura, 2014 & Chile & 200 & $\mathrm{E}$ \\
8 & Deshpande et al., 2015 & India & 130 & $\mathrm{O}$ \\
9 & Al-amad et al., 2016 & Emiratos Árabes & 350 & $\mathrm{O}$ \\
10 & Bodrumlu et al., 2016 & Turquía & 248 & $\mathrm{E}$ \\
11 & Brattabø et al., 2016 & Noruega & 1200 & O y A \\
12 & Mogaddam et al., 2016 & Arabia Saudita & 208 & O \\
13 & Uldum et al., 2017 & Dinamarca & 964 & O y A \\
14 & Olatosi et al., 2018 & Nigeria & 179 & O \\
15 & Brattabø et al., 2018 & Noruega & 1200 & O y A \\
E: estudiantes / O: odontólogos / A: asistentes o higienitas dentales & & \\
\hline
\end{tabular}


CIFUENTES, H. C.; CONTRERAS, R. C.; TORRES, M. A. \& GONZALES, A. S. Maltrato infantil: conocimiento y actitud del cirujano dentista. Marco legal y revisión de la literatura. Int. J. Odontostomat., 14(2):160-166, 2020.

Tabla II. Análisis bibliográfico (parte 2).

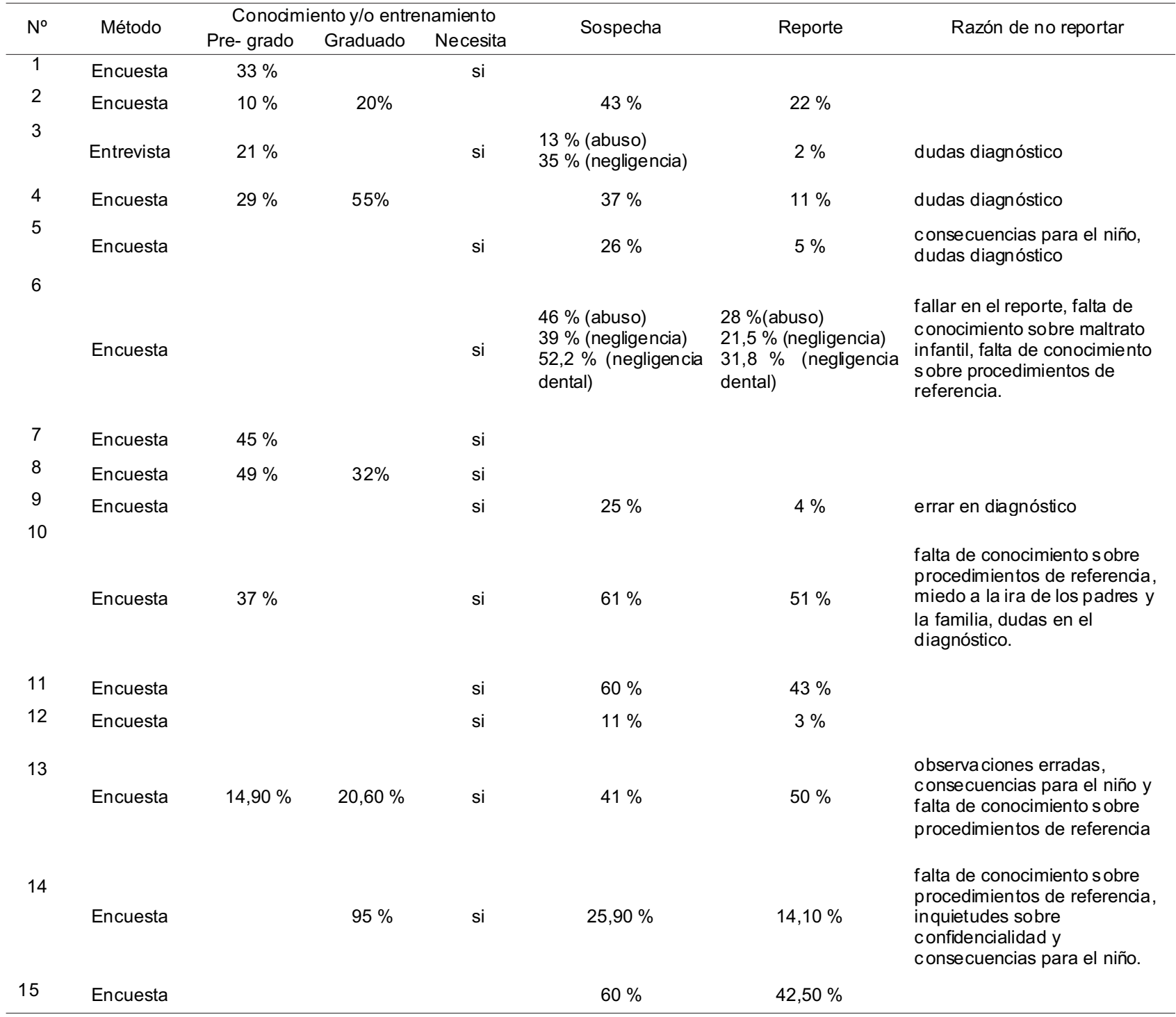

\section{DISCUSIÓN}

El abuso infantil puede ser físico, sexual, psicológico o abandono. Las lesiones asociadas pueden presentarse en múltiples formas, como hematomas, quemaduras, laceraciones, cortes, equimosis, contusiones y abrasiones en el cuerpo, la cara, los labios y la boca. En ella, la agresión física puede ser identificada por traumatismos dentales y lesiones en los tejidos blandos. La situación de abandono dental corresponde a un tipo de negligencia y es por la falta voluntaria de los padres o tutores para buscar y seguir con el tratamiento necesario para asegurar un nivel de salud oral esencial para una función adecuada, libre de dolor y de infección.

La Academia Americana del Comité Pediátrico del Abuso y Abandono infantil, la Academia Americana de Odontopediatría y el Ministerio de Salud de Chile (2014) indican que se debe prestar especial atención a señales de alerta como quemaduras (cigarro), alopecia traumática y a signos de abandono en ropa, falta de higiene, desnutrición y focos de infección por caries. 
Fierro et al. (2012) señalan que la negligencia es la forma más común de maltrato y corresponde al incumplimiento de los padres en buscar y seguir adelante con el tratamiento necesario para asegurar la salud oral de sus hijos. Una madre o padre negligente es aquel que después de haber sido advertido por el profesional de la importancia de la salud oral no lleva a su hijo a los controles periódicos con el cirujano dentista.

Es lamentable que en la Guía Clínica de detección y primera respuesta a niños, niñas y adolescentes víctimas de maltrato por parte de familiares o cuidadores del Ministerio de Salud de Chile (2013), que debería estar enfocada para todo el equipo de salud, solo se mencione las lesiones bucales al evaluar las mucosas y que en ningún momento se considere la presencia de caries de gran extensión o múltiples lesiones como señal de alerta de negligencias o abandono. De todas maneras entregan flujogramas e instrucciones claras de apertura de la situación de maltrato, medidas de protección, evaluación de riesgo, denuncia, derivación asistida, seguimiento y coordinación intersectorial; procedimientos con los que el equipo de salud oral debería estar más empoderado.

La Norma para la supervisión de niños y niñas de 0 a 9 años en atención primaria, del Ministerio de Salud de Chile (2014), es un documento completo e integral, ya que aborda la atención de los niños en todos los ámbitos y por todo el equipo de Salud, y es responsabilidad de los(as) odontólogos(as) el estar al tanto de su contenido.

Llama mucho la atención el hecho de que en la Guía de atención primaria odontológica del preescolar de 2 a 5 años del Ministerio de Salud de Chile (2009) solo se menciona una vez el maltrato o abuso infantil, considerando el contacto regular que los odontólogos tienen con los niños y sus familias; y que las lesiones cráneo-faciales y de cuello ocurren en más de la mitad de los casos de maltrato infantil. Una probable razón de esta limitante es que la guía es muy antigua y la inclusión de odontólogos(as) al modelo de salud familiar y atención multidisciplinaria en salud es más bien reciente. Se espera que en la actualización de esta guía se consideren y plasmen las señales de alerta de maltrato y abuso infantil, así como los flujogramas de denuncia de los casos.

En relación al conocimiento o información en aspectos de maltrato o abuso infantil en Chile, Fierro et al. afirman que $50,9 \%$ de sus encuestados declara haber recibido información sobre maltrato infantil ya sea de fuentes formales como informales. Con respecto a la actitud, 23 (43\%) odontólogos reconocen haber sospechado alguna vez algún caso de maltrato infantil, de ellos 16 (70 \%) fueron mujeres y 7 (30\%) fueron hombres. De esos 23 odontólogos sólo 13 (57\%) han denunciado sus sospechas, de ellos 10 $(77 \%)$ fueron mujeres y $3(23 \%)$ fueron hombres. Brattabø et al. (2016), reporta medias de 0,69 y 0,54 $(p<, 05)$ en el reporte de mujeres y hombres respectivamente, es decir que al igual que lo observado por Fierro et al. son las mujeres quienes realizan más denuncias de sus sospechas.

En cuanto al conocimiento de los estudiantes Asfura (2014) describe que el $45 \%$ de los estudiantes de odontología, conoce del tema. Bodrumlu et al. indican que ante la pregunta "¿Alguna vez tuvo capacitación sobre abuso infantil?" 2,17 \% de tercer año, $13,27 \%$ de cuarto año y $21,43 \%$ de estudiantes de quinto año respondieron positivamente. Estos autores evaluaron también el conocimiento sobre los aspectos legales referentes al tema y la proporción de estudiantes que respondieron que sabían que la leyde su país ordena a los dentistas que denuncien casos de abuso infantil es $43,1 \%, 58,41 \%$ y $90,48 \%$ para estudiantes de tercer, cuarto y quinto año, respectivamente, dato muy relevante e interesante que deja la duda de cual será la realidad en Chile.

Al-Amad et al. (2016), Cucovic-Bagic et al. (2013), Harris et al. (2013) y Laud et al. (2013) en estudios realizados en varios países informaron de las dificultades de los dentistas en el diagnóstico, la documentación y la denuncia de casos de sospecha de abuso a las autoridades, también observan que el porcentaje de sospechas es mayor al de reportes, en promedio $35 \%$ y $24 \%$ respectivamente, y que las principales razones de no reportar son: dudas en el diagnósticos, temor de las consecuencias para el niño y falta de conocimiento en los procedimientos de referencia.

En cuanto a la necesidad de capacitación al respecto, todos los estudios concuerdan en el hecho de que se necesita formar más profundamente al respecto, tanto en la detección de los signos y síntomas de alerta, en como afrontar la situación y cuales son los protocolos o acciones a seguir en cuanto a reporte y derivación (Fierro et al.; Laud et al.; Harris et al.; Asfura; Tilvawala et al., 2014; Deshpande et al., 2015; Al-Amad et al.; Mogaddam et al., 2016; Brattabø et al., 2016, 2018; Olatosi et al., 2018). 
Con los antecedentes expuestos queda claro que la falta de seguridad para realizar las denuncias es principalmente debido a un pobre conocimiento en el tema, que se puede deber al hecho de que la mayoría de las disciplinas de salud no incluye en sus planes de estudio ni en los programas de educación continua aspectos de educación y formación relacionados con la detección de signos y síntomas de alerta, ni el abordaje del maltrato o abuso infantil (Fierro et al.), por lo tanto los profesionales no están preparados para detectar ni ofrecer solución real a sus sospechas, lo que puede estar generando un subregistro y dejando sin ayuda o atención a las víctimas.

Cucovic-Bagic et al. indican que el 91,96 \% de sus encuestados declaran que se debería tocar la temática de protección infantil en los programas de pregrado. En ese contexto es importante tener presente que en países como Noruega las instituciones de educación superior que imparten odontología han incluido el maltrato infantil y los protocolos de notificación obligatoria en sus programas de estudios (Brattabø et al., 2018), ejemplo que las casas de estudio que imparten la carrera de odontología en Chile deberían seguir.

\section{CONCLUSIONES}

La participación del equipo de salud oral en la denuncia de maltrato o abuso infantil es pobre o insuficiente tanto en Chile como en el resto del mundo y la principal razón de ello es la inseguridad para abordar el tema por falta de conocimiento tanto de los signos y síntomas de alerta, como de los procedimientos administrativos para realizar las denuncias.

Dada la importancia del tema, el papel del (la) cirujano dentista al trabajar directamente con niños(as), y el aumento en el reporte de casos sospechosos de violencia, se sugiere mejorar las políticas públicas para la protección integral de los menores, lo cual debe incluir guías clínicas que integren a los odontólogos(as) en su elaboración.

Por otro lado se propone incorporar el tema en la malla curricular de estudiantes de pregrado, incluyendo como mínimo herramientas para detección de signos y síntomas clave de maltrato y abuso infantil tanto en el territorio maxilofacial como en el análisis conductual de los menores, como afrontar la situación y cuáles son los protocolos o acciones a seguir en cuanto a reporte y derivación, cual es el marco legal y organismos gubernamentales encargados.

Además se propone fuertemente realizar una vigilancia respecto a conocimiento y actitudes entre los odontólogos en Chile y de ser necesario, incluir en los programas de capacitación anual de servicios públicos de salud cursos de formación en aspectos de: detección de signos y síntomas clave de maltrato y abuso infantil tanto en el territorio maxilofacial como en el análisis conductual de los menores, también cuales son los procedimientos administrativos para realizar una denuncia y el marco legal de Chile con respecto a funcionarios públicos.

Finalmente, se debe recordar que es responsabilidad de cada ciudadano y profesional de la salud, el estar al tanto de las leyes que aplican a la profesión, y en su justa medida tener el conocimiento para poder cumplirlas.

CIFUENTES, H. C.; CONTRERAS, R. C.; TORRES, M. A. \& GONZALES, A. S. Child abuse: Knowledge and attitude of the dentist, legal framework and review of the literature. Int. J. Odontostomat., 14(2):160-166, 2020.

ABSTRACT: Abuse and neglect of children constitutes a social phenomenon that unfortunately is spread throughout the world without ethnic, geographical or social context barriers. In Chile, until 2012, $71 \%$ of children suffered some type of abuse within their family. Skull-facial and neck injuries occur in more than half of the cases. The Dentist has a legal, moral and ethical obligation as a citizen and health professional to notify the authorities of suspected cases of abuse. The objective of the study was to evaluate the extent of the dental surgeons' knowledge regarding child abuse and their attitude towards the detection and reporting of child abuse. Bibliographic search in PUBMED using keywords: "child abuse" and "dentistry". In the study, 132 articles were found, of which 15 were full text. Studies in several countries reported the difficulties of dentists in the diagnosis, documentation and reporting of cases of suspected abuse to the authorities, in addition to the need for further training in the subject. Given the importance of this issue, the role of the dentist working directly with children and adolescents, and the increase in the reporting of suspected cases of violence, it is strongly suggested to monitor the knowledge and attitudes among dentists in Chile.

KEY WORDS: child abuse, dentistry, legal aspects. 


\section{REFERENCIAS BIBLIOGRÁFICAS}

Al-Amad, S. H.; Awad, M. A.; Al-Farsi, L. H. \& Elkhaled, R. H. Reporting child abuse cases by dentists working in the United Arab Emirates (UAE). J. Forensic Leg. Med., 40:12-5, 2016.

American Academy of Pediatrics Committee on Child Abuse and Neglect and the American Academy of Pediatric Dentistry. Guideline on Oral and Dental Aspects of Child Abuse and Neglect. Clinical Guidelines, Reference Manual, 32(6):137-40, 2010.

Asfura, H. B.; Lecannelier, B. F.; Schovelin, A. \& Silva, J. Conocimientos y actitudes de estudiantes de odontología frente al maltrato infantil, Universidad del Desarrollo, Concepción, 2014. Rev. Soc. Chil. Odontopediatr., 29(2):16, 2014

Brattabø, I. V.; Bjarknes, R. \& Ãstram, A. N. Reasons for reported suspicion of child maltreatment and responses from the child welfare - a cross-sectional study of Norwegian public dental health personnel. BMC Oral Health, 18(1), 2018.

Brattabø, I. V.; Iversen, A. C.; Åstrøm, A. N. \& Bjørknes, R. Experience with suspecting child maltreatment in the Norwegian Public Dental Health Services, a national survey. Acta Odontol. Scand., 74(8):626-32, 2016

Comité Operativo de Intervención en Maltrato Infantil del Hospital Roberto del Río; Galaz, L. M. I.; Figueroa, E. D.; Orellana, C. C.; Henríquez, M. E.; Galdames, F. A. M.; Lagos, S. V. \& Schneider, R. M. Guía Clínica Maltrato Infantil. Santiago de Chile, Servicio de Salud Metropolitano Norte, 2010.

Cucovic-Bagic, I.; Welbury, R. R.; Flander, G. B.; Hatibovic-Kofman, S. \& Nuzzolese, E. Child protection: legal and ethical obligation regarding the report of child abuse in four different countries. $J$. Forensic Odontostomatol., 31(1):15-21, 2013.

Deshpande, A.; Macwan, C.; Poonacha, K. S.; Bargale, S.; Dhillon, S. \& Porwal, P. Knowledge and attitude in regards to physical child abuse amongst medical and dental residents of Central Gujarat: a cross-sectional survey. J. Indian Soc. Pedod. Prev. Dent., 33(3):177-82, 2015.

Fierro, M. C.; Salazar, S. E.; Ruiz, S. L.; Luengo, M. L. \& Pérez, F. A. Attitude towards child abuse according to the knowledge of dentists in Concepción, Chile. Int. J. Odontostomat, 6(1):10510, 2012.

Harris, C. M.; Welbury, R. \& Cairns, A. M. The scottish dental practitioner's role in managing child abuse and neglect. Br. Dent. J., 214(9):E24, 2013.

Krug, E. G.; Dahlberg, L. L.; Mercy, J. A.; Zwi, A. B. \& Lozano, R. (Eds.). World Report on Violence and Health. Geneva, World Health Organization, 2002. Disponible en: https://apps.who.int/ i ris/bitstrea m/h a nd le / $10665 / 42495 /$ 9241545615_eng.pdf?sequence $=1$

Laud, A.; Gizani, S.; Maragkou, S.; Welbury, R. \& Papagiannoulis, L. Child Protection Training, Experience, and Personal Views of Dentists in the Prefecture of Attica, Greece. Int. J. Paediatr. Dent., 23(1):64-71, 2013.

Ministerio de Desarrollo Social. Primer Informe sobre los Avances en el Ámbito de la Protección y Cumplimiento de los Derechos de la Infancia y de la Adolescencia. Santiago de Chile, Consejo de Ministros para la Infancia y Adolescencia, Ministerio de Desarrollo Social, Gobierno de Chile, 2003.

Ministerio de Hacienda. Ley 18834. Sobre Estatuto Administrativo. Santiago de Chile, Biblioteca del Congreso Nacional de Chile, 2005. Disponible en: https://www.leychile.cl/ Navegar?idNorma $=236392$

Ministerio de Justicia. Código Penal. Santiago de Chile, Biblioteca del Congreso Nacional de Chile, 1874. Disponible en: https:// www.leychile.cl/Navegar?idNorma $=1984$
Ministerio de Justicia. Ley 19696. Código Procesal Penal. Santiago de Chile, Biblioteca del Congreso Nacional de Chile, 2000. Disponible en: https://www.leychile.cl/Navegar?idNorma=176595

Ministerio de Salud. Guía Clínica: Detección y Primera Respuesta a Niños, Niñas y Adolescentes Víctimas de Maltrato por parte de Familiares o Cuidadores. Santiago de Chile, Ministerio de Salud, Gobierno de Chile, 2013.

Ministerio de Salud. Guía Clínica. Atención Primaria Odontológica del Preescolar de 2 a 5 Años. Santiago de Chile, Ministerio de Salud, Gobierno de Chile, 2009.

Ministerio de Salud. Norma Técnica para la Supervisión de Niños y Niñas de 0 a 9 años en la Atención Primaria de Salud. Santiago de Chile, Ministerio de Salud, Gobierno de Chile, 2014.

Mogaddam, M.; Kamal, I.; Merdad, L. \& Alamoudi, N. Knowledge, attitudes, and behaviors of dentists regarding child physical abuse in Jeddah, Saudi Arabia. Child Abus. Negl., 54:43-56, 2016.

Olatosi, O. O.; Ogordi, P. U.; Oredugba, F. A. \& Sote, E. O. Experience and knowledge of child abuse and neglect: a survey among a group of resident doctors in Nigeria. Niger. Postgrad. Med. J., 25(4):225-33, 2018

Tilvawala, D.; Murray, C.; Farah, R. \& Broadbent, J. M. New Zealand dental therapists' beliefs regarding child maltreatment. Aust. $N$. Z. J. Public Health, 38(5):480-4, 2014.

UNICEF. $4^{\circ}$ Estudio de Maltrato Infantil en Chile. Análisis Comparativo 1994 - 2000 - 2006 - 2012. Santiago de Chile, Fondo de las Naciones Unidas para la Infancia, UNICEF, 2015.

Dirección para correspondencia:

Carla Cifuentes Harris

Residente Especialidad Odontología Legal y Forense

Universidad de Chile

Santiago

CHILE

Email: carla.cifuentes.harris@gmail.com

Recibido : 13-09-2019

Aceptado: 18-10-2019 